\title{
Specificity of dermal mucin in the diagnosis of lupus erythematosus: comparison with other dermatitides and normal skin ${ }^{\dagger}$
}

Increased dermal mucin is a feature of lupus erythematosus (LE); however, its amount and distribution have not been well characterized. The differentiation of LE from other forms of dermatitis can be challenging when other features of LE are subtle or equivocal. One hundred and thirty-five skin specimens showing LE, graft vs. host disease, erythema multiforme/fixed drug eruption, lichen planus, polymorphous light eruption (PMLE), urticaria, eczematous dermatitis and psoriasis and normal skin with and without photodamage were collected. The amounts of mucin in the papillary, superficial reticular and deep reticular dermis were scored from 0 to 3 on hematoxylin-eosin $(\mathrm{H} \& \mathrm{E})$ and alcian blue $(\mathrm{AB})$ stains, and compared between groups. The mean scores in the reticular dermis were significantly higher in LE than in other categories except PMLE and eczematous dermatitis. A combined $\mathrm{H} \& \mathrm{E}+\mathrm{AB}$ score of $\geq 5$ in the superficial reticular dermis gave an overall specificity of $85.7 \%$ for LE. Mucin in the papillary dermis failed to distinguish among entities. Normal photodamaged skin showed significantly more mucin in the superficial reticular dermis compared to non-photodamaged skin. While LE is associated with increased mucin deposition, scant to moderate amount of mucin alone has limited specificity and is common in other dermatitides or photodamaged skin.

Keywords: lupus erythematosus, mucin, photodamage, polymorphous light eruption, tumid

Vincent JG, Chan MP. Specificity of dermal mucin in the diagnosis of lupus erythematosus: comparison with other dermatitides and normal skin.

J Cutan Pathol 2015; 42: 722-729. (C) 2015 John Wiley \& Sons A/S. Published by John Wiley \& Sons Ltd

\section{Jeremy G. Vincent ${ }^{1}$ and May P. Chan ${ }^{1,2}$}

${ }^{1}$ Department of Pathology, University of Michigan, Ann Arbor, MI, USA and ${ }^{2}$ Department of Dermatology, University of Michigan, Ann Arbor, MI, USA

$\dagger$ Presented in part at the $103^{\text {rd }}$ Annual Meeting of the United States and Canadian Academy of Pathology, San Diego, CA, on March 4, 2014.
May P. Chan, MD,

Department of Pathology, University of Michigan, 1301 Catherine Street, Medical Science I, M3261, Ann Arbor, Ml 48109-5602, USA Tel: +17347644460 ,

Fax: +1 7347644690

e-mail: mpchan@med.umich.edu

Accepted for publication March 15, 2015
Increased dermal mucin is one of the classic features of cutaneous lupus erythematosus (LE); however, the amount and the distribution of mucin have not been well characterized. Differentiation of LE from other inflammatory dermatitides can be challenging when scant to moderate amount of dermal mucin is present, but other classic features of LE are subtle or equivocal.

Increased dermal mucin and superficial to deep perivascular and periadnexal lymphocytic infiltrate are fairly constant histopathologic 
features of cutaneous LE. ${ }^{1,2}$ Additional findings such as interface dermatitis, basement membrane thickening and follicular plugging are seen at varying frequencies depending on the specific variant and chronicity of cutaneous LE. ${ }^{3-6}$ While the diagnosis of LE is straightforward when all of the above histopathologic features are present in conjunction with classic clinical presentation, diagnostic difficulty arises when clinical information is limited and when only few classic features are observed histopathologically. For example, other interface or perivascular dermatitides may present with variable amount of dermal mucin and thus closely mimic cutaneous LE. It also remains unclear whether chronic sun exposure may contribute to mucin deposition to a certain degree. To our knowledge, study on the amount of dermal mucin in these non-LE conditions is lacking in the current literature.

Our objective was to address the above diagnostic challenge by comparing the quantity and the distribution of dermal mucin in cutaneous LE to a variety of interface, perivascular, spongiotic and psoriasiform dermatitides as well as normal skin without dermatitis. We also aimed to study the effect of chronic sun exposure on mucin deposition by comparing normal skin with and without solar elastosis.

\section{Materials and methods}

After approval by the Institutional Review Board, the surgical pathology database at University of Michigan was searched for 'lupus erythematosus', 'tumid lupus', 'discoid lupus', 'subacute cutaneous lupus', 'systemic lupus', 'eczema', 'psoriasis', 'lichen planus' (LP), 'graft vs. host disease' (GVHD), 'erythema multiforme' (EM), 'fixed drug eruption' (FDE), 'urticaria' and 'polymorphous light eruption' (PMLE) between years 2010 and 2014. All pathologic slides were retrospectively reviewed and correlated with clinical data (obtained from requisition form and/or electronic medical record) to confirm the original diagnoses. Cases with diagnostic uncertainty were eliminated. 'Eczematous dermatitis' refers to cases showing a primarily spongiotic pattern, including atopic dermatitis, contact dermatitis, nummular dermatitis and eczematous drug reaction. Normal skin samples with and without evidence of photodamage (solar elastosis) were obtained from the tip margins of wide local excisions performed for melanoma and Merkel cell carcinoma.
Both hematoxylin-eosin (H\&E)- and alcian blue $(A B)$-stained sections were evaluated for dermal mucin in three different compartments: papillary dermis, superficial reticular dermis and deep reticular dermis. The amount of mucin in each compartment was scored separately on $\mathrm{H} \& \mathrm{E}$ and $\mathrm{AB}$ as follows: 0 , absent; 1 , scant wisps of mucin that are barely noticeable; 2 , moderate amount of mucin almost filling the spaces between collagen fibers; or 3 , abundant mucin pools filling and expanding the spaces between collagen fibers. The $\mathrm{H} \& \mathrm{E}$ and $\mathrm{AB}$ scores in each compartment were then combined to give a total score of $0-6$. Four selected cases were also stained with colloidal iron for comparison. Chi-square and two-tailed $t$-tests were performed between groups. A $p$-value of $<0.05$ was considered statistically significant. The specificities and sensitivities of dermal mucin for the diagnosis of LE were calculated using different cut-off combined scores.

\section{Results}

A total of 135 skin samples, including cutaneous LE $(n=35)$, GVHD $(n=8), E M / F D E ~(n=9)$, LP $(n=15)$, PMLE $(n=11)$, urticaria $(n=8)$, eczematous dermatitis $(n=8)$, psoriasis $(n=11)$, normal skin with solar elastosis $(n=16)$ and normal skin without solar elastosis $(n=14)$ were selected. The LE cases included discoid $(n=8)$, subacute cutaneous $(n=7)$, systemic $(n=4)$, tumid $(\mathrm{n}=13)$ and not-otherwise-specified $(n=3)$ subtypes. None of the patients in the non-LE groups had known history of LE.

The mean combined $(\mathrm{H} \& \mathrm{E}+\mathrm{AB})$ mucin scores for all entities are summarized in Table 1 . Four specimens were superficial and were excluded from the calculations for the deep reticular dermal compartment. Sixteen cases $(11.9 \%)$ had a discrepancy of at least two score points between $\mathrm{H} \& \mathrm{E}$ and $\mathrm{AB}$ in at least one compartment. The $p$-values obtained from two-tailed $t$-tests between groups are shown in Table 2 . Significantly more mucin was present in both superficial and deep reticular dermis in LE when compared to GVHD, EM/FDE, LP, urticaria, psoriasis and normal skin (Fig. 1). However, no significant difference was found when comparing LE with PMLE and eczematous dermatitis in all three compartments (Figs 2 and 3). Mucin in the papillary dermis failed to distinguish LE from other entities. Normal photodamaged skin (with solar elastosis) showed significantly more mucin in the superficial reticular dermis compared to normal non-photodamaged skin 
Table 1. Mean combined $(H \& E+A B)$ mucin scores

\begin{tabular}{lcccc}
\hline Condition & Papillary & $\begin{array}{c}\text { Superficial } \\
\text { reticular } \\
\text { dermis }\end{array}$ & $\begin{array}{c}\text { Deep } \\
\text { reticular } \\
\text { dermis }\end{array}$ \\
\hline LE (all subtypes) & 35 & 3.17 & 4.57 & 2.83 \\
$\quad$ DLE & 8 & 2.88 & 4.25 & 2.38 \\
$\quad$ SCLE & 7 & 3.71 & 4.71 & 2.57 \\
$\quad$ SLE & 4 & 3.00 & 3.75 & 2.25 \\
$\quad$ TLE & 13 & 3.15 & 4.92 & 3.38 \\
$\quad$ NOS & 3 & 3.00 & 4.67 & 3.00 \\
GVHD & 8 & 4.00 & 2.50 & 1.13 \\
EM/FDE & 9 & 2.89 & 2.67 & 1.22 \\
LP & 15 & 3.13 & 2.27 & 0.75 \\
PMLE & 11 & 3.55 & 4.09 & 2.64 \\
Urticaria & 8 & 3.13 & 3.38 & 1.75 \\
Eczematous & 8 & 2.88 & 3.50 & 2.00 \\
Psoriasis & 11 & 3.18 & 3.18 & 1.20 \\
Normal, & 16 & 3.13 & 3.06 & 1.44 \\
$\quad$ photodamaged & & & & \\
Normal, & 14 & 2.71 & 2.07 & 0.86 \\
$\quad$ non-photodamaged & & & & \\
\hline
\end{tabular}

$A B$, Alcian blue; DLE, discoid lupus erythematosus; EM, erythema multiforme; FDE, fixed drug eruption; GVHD, graft vs. host disease; H\&E, hematoxylin-eosin; LE, lupus erythematosus; LP, lichen planus; n, number of cases; NOS, not-otherwise-specified; PMLE, polymorphous light eruption; SCLE, subacute cutaneous lupus erythematosus; SLE, systemic lupus erythematosus; TLE, tumid lupus erythematosus.

(without solar elastosis) $(p=0.0140)$; a similar difference was also observed with $\mathrm{AB}$ scores alone $(p=0.0007)$ (Fig. 4). Other than LE and PMLE, none of the entities showed significant differences in the amount of superficial reticular dermal mucin compared to normal skin.

The sensitivities and the specificities of reticular dermal mucin for LE against other dermatitides are listed in Table 3. Compared to the superficial reticular dermis, mucin in the deep reticular dermis yielded the highest specificities and the lowest sensitivities. Similarly, using a higher combined score ( 5 and above) resulted in higher specificities and lower sensitivities compared to a lower cut-off ( 4 and above).

\section{Discussion}

Although the distinction between LE and other inflammatory dermatitides in the setting of scant to moderate amount of dermal mucin presents a diagnostic dilemma that is not uncommon, it has drawn little attention in the literature. To address this issue, we performed a retrospective analysis on confirmed cases of LE, GVHD, EM/FDE, LP, PMLE, urticaria, eczematous dermatitis and psoriasis to characterize the amount and the
Table 2. Comparison of combined $(H \& E+A B)$ mucin scores ( $p$-values from two-tailed $t$-tests)

\begin{tabular}{|c|c|c|c|}
\hline & $\begin{array}{c}\text { Papillary } \\
\text { dermis }\end{array}$ & $\begin{array}{l}\text { Superficial } \\
\text { reticular } \\
\text { dermis }\end{array}$ & $\begin{array}{c}\text { Deep reticular } \\
\text { dermis }\end{array}$ \\
\hline LE vs. GVHD & 0.1353 & 0.0015 & 0.0027 \\
\hline LE vs. EM/FDE & 0.5910 & 0.0009 & 0.0019 \\
\hline LE vs. LP & 0.9324 & $<0.0001$ & $<0.0001$ \\
\hline LE vs. PMLE & 0.4670 & 0.3240 & 0.6733 \\
\hline LE vs. urticaria & 0.9359 & 0.0417 & 0.0476 \\
\hline LE vs. eczematous & 0.5997 & 0.0580 & 0.1256 \\
\hline LE vs. psoriasis & 0.9824 & 0.0074 & 0.0019 \\
\hline $\begin{array}{l}\text { Photodamaged normal vs. } \\
\text { non-photodamaged } \\
\text { normal }\end{array}$ & 0.2413 & 0.0140 & 0.0821 \\
\hline LE vs. normal* & 0.4383 & $<0.0001$ & $<0.0001$ \\
\hline GVHD vs. normal ${ }^{*}$ & 0.0109 & 0.8506 & 0.9136 \\
\hline EM/FDE vs. normal* & 0.9096 & 0.8799 & 0.8668 \\
\hline LP vs. normal* & 0.5876 & 0.3438 & 0.1960 \\
\hline PMLE vs. normal* & 0.1429 & 0.0006 & $<0.0001$ \\
\hline Urticaria vs. normal* & 0.6683 & 0.1107 & 0.1268 \\
\hline Eczematous vs. normal* & 0.8912 & 0.0510 & 0.0331 \\
\hline Psoriasis vs. normal ${ }^{*}$ & 0.4765 & 0.1718 & 0.9275 \\
\hline
\end{tabular}

$A B$, Alcian blue, EM, erythema multiforme; FDE, fixed drug eruption; GVHD, graft vs. host disease; H\&E, hematoxylin-eosin; LE, lupus erythematosus (all subtypes); LP, lichen planus; PMLE, polymorphous light eruption.

Boldface indicates statistical significance $(p<0.05)$.

${ }^{*}$ All normal cases with and without photodamage.

location of dermal mucin in these entities. We hypothesized that increased dermal mucin may be seen in a variety of dermatitides, and therefore has limited specificity for LE in the absence of other classic features.

Dermal mucin is composed of various glycosaminoglycans, which are long polymers that are polyanionic and contain repeating disaccharide units. Glycosaminoglycans can be divided into six major groups, including chondroitin sulfate, dermatan sulfate, heparin, heparin sulfate, hyaluronan and keratan sulfate. ${ }^{7,8}$ Depending on the specific type, glycosaminoglycans play a variety of roles including cell-cell interactions, absorbing water and binding to extracellular protein. ${ }^{9-13}$ Additionally, there is some evidence to suggest that glycosaminoglycans function immunologically by activating macrophages, dendritic cells and neutrophils, and may inhibit the effects of tumor necrosis factor-alpha and interleukin-6. ${ }^{14-16}$ It has been shown that incubation of normal fibroblasts with serum from a patient with LE induced production of glycosaminoglycans. ${ }^{17}$ While the exact mechanism is poorly understood, it is 

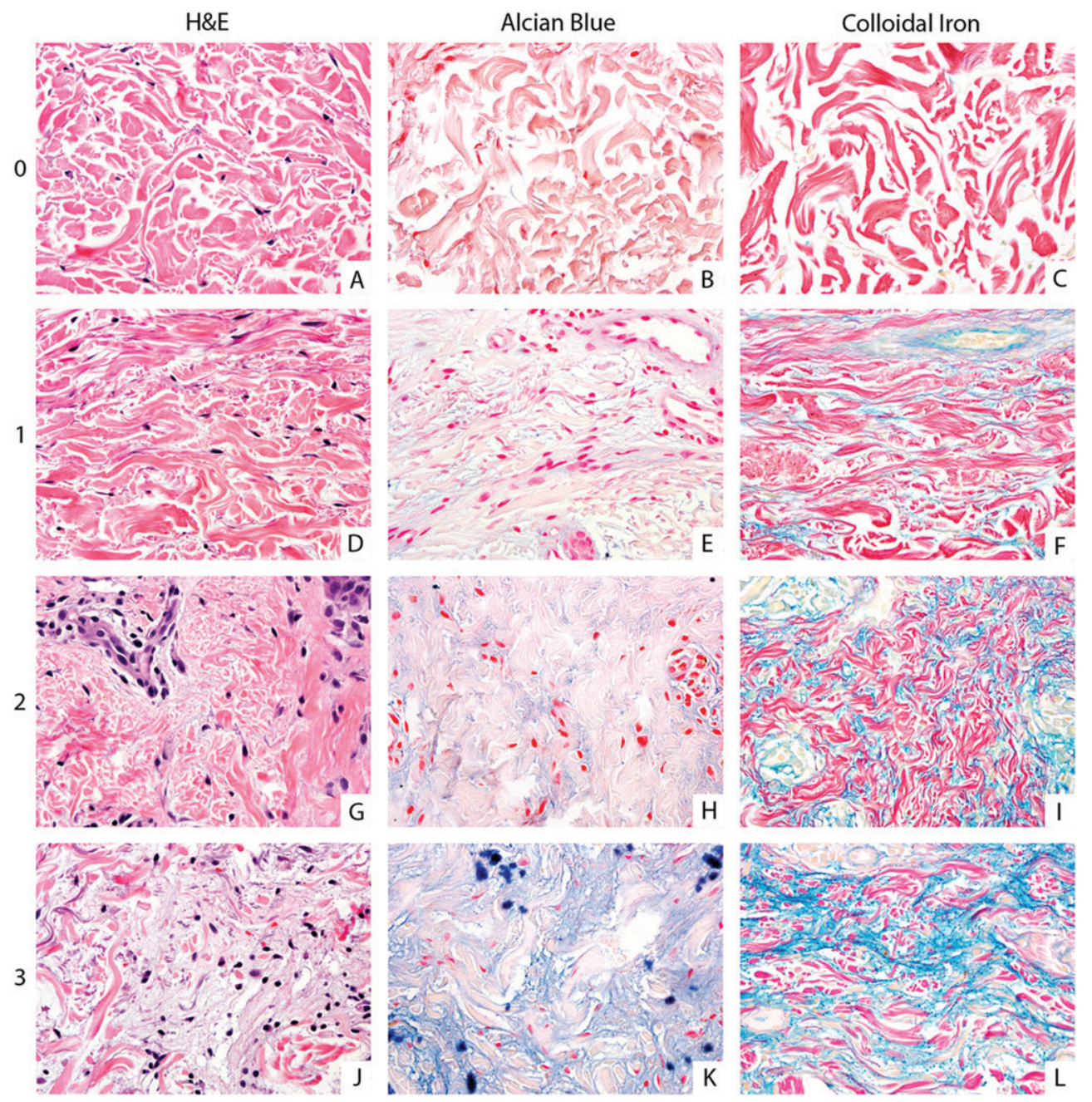

Fig. 1. Scoring of dermal mucin on hematoxylin-eosin $(\mathrm{H} \& \mathrm{E})$ and alcian blue $(\mathrm{AB})$ stains. Colloidal iron stain was also performed on these selected cases for comparison. Mucin is appreciated as a blue-gray, beaded, stringy material in between and attached to collagen fibers on $\mathrm{H} \& \mathrm{E}$, which gives a blue color on $\mathrm{AB}$ and colloidal iron stains. A, B and C) An example of graft vs. host disease without mucin (combined score $=0$ ). D, E and F) An example of lichen planus with scant wisps of mucin barely noticeable on $\mathrm{H} \& \mathrm{E}$ and $\mathrm{AB}$ stains (combined score $=1+1=2$ ). Note an apparently greater amount of mucin is appreciable in a patchy fashion on colloidal iron stain. G, H and I) An example of erythema multiforme with moderate amount of mucin almost filling the spaces between collagen fibers (combined score $=2+2=4$ ). Again, more intense staining is noted on colloidal iron compared to $\mathrm{AB}$, with the former showing diffuse mucin deposition between collagen fibers. J, K and L) An example of tumid lupus erythematosus with abundant mucin filling and expanding the spaces between collagen fibers (combined score $=3+3=6)$. Similar findings are observed on colloid iron stain. [H\&E (A, D, G and J), AB (B, E, H and K) and colloidal iron (C, F, I and L), original magnifications $\times 400]$

thought that mucin accumulation is predominantly driven by fibroblast and endothelial cell production or decreased degradation. ${ }^{18,19}$ It is therefore not surprising that a variety of inflammatory skin diseases may also display increased dermal mucin.

We evaluated both conventional and $\mathrm{AB}$ stains in order to attempt to provide more specific and objective scores on the quantification of mucin, although similar trends were observed on either $\mathrm{H} \& \mathrm{E}$ or $\mathrm{AB}$ alone. Discrepancies of at least two score points between $\mathrm{H} \& \mathrm{E}$ and $\mathrm{AB}$ were observed in only a small subset $(11.9 \%)$ of cases, which may be attributable to slight differences in tissue processing and staining. It is also noteworthy that colloidal iron stain, another special stain commonly used to highlight dermal mucin, tends to show more robust staining. As illustrated in Fig. 1, assessment of scores should be adjusted accordingly when colloidal stain is used instead of $\mathrm{AB}$. By dividing the dermis into three compartments, we found that papillary dermal mucin was a frequent finding across different entities including normal skin, and provided no diagnostic value in distinguishing LE from other conditions examined in this 


\section{Vincent \& Chan}
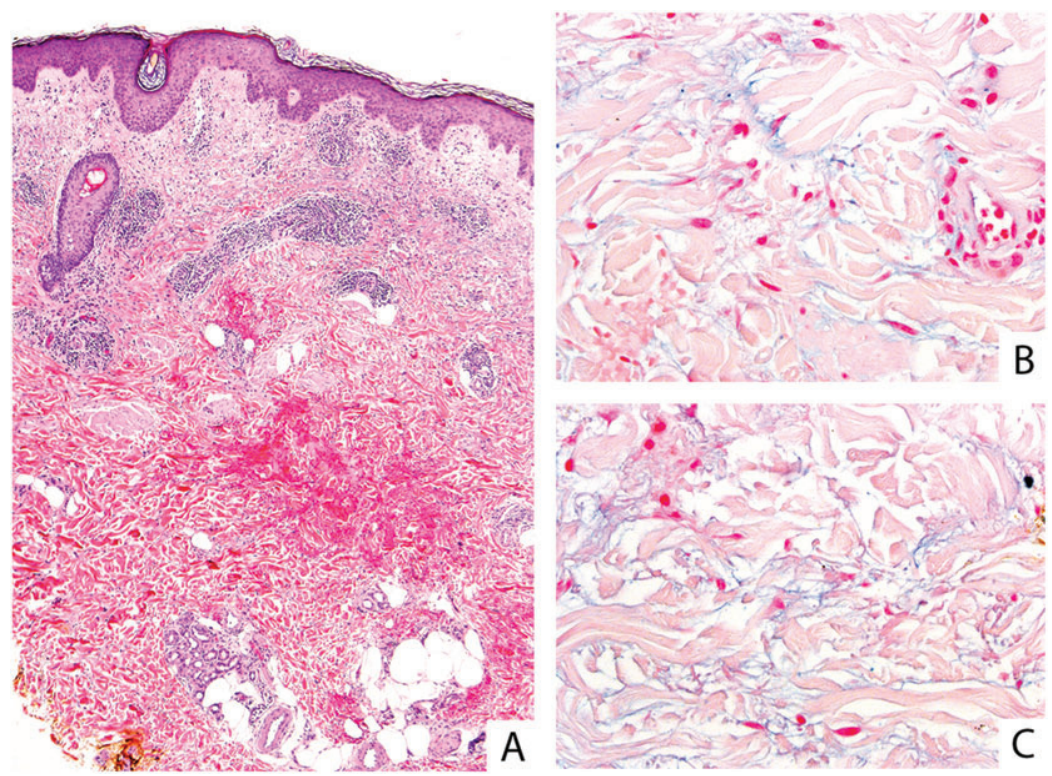

Fig. 2. An example case of polymorphous light eruption. A) Low magnification shows a superficial to mid dermal perivascular lymphocytic infiltrate with mild papillary dermal edema, closely mimicking tumid lupus erythematosus. Moderate amount of mucin is present in both $(B)$ superficial reticular dermis and $(\mathrm{C})$ deep reticular dermis. [hematoxylin-eosin, original magnification $\times 40$ (A); alcian blue, original magnifications $\times 400$ (B and $\mathrm{C})]$
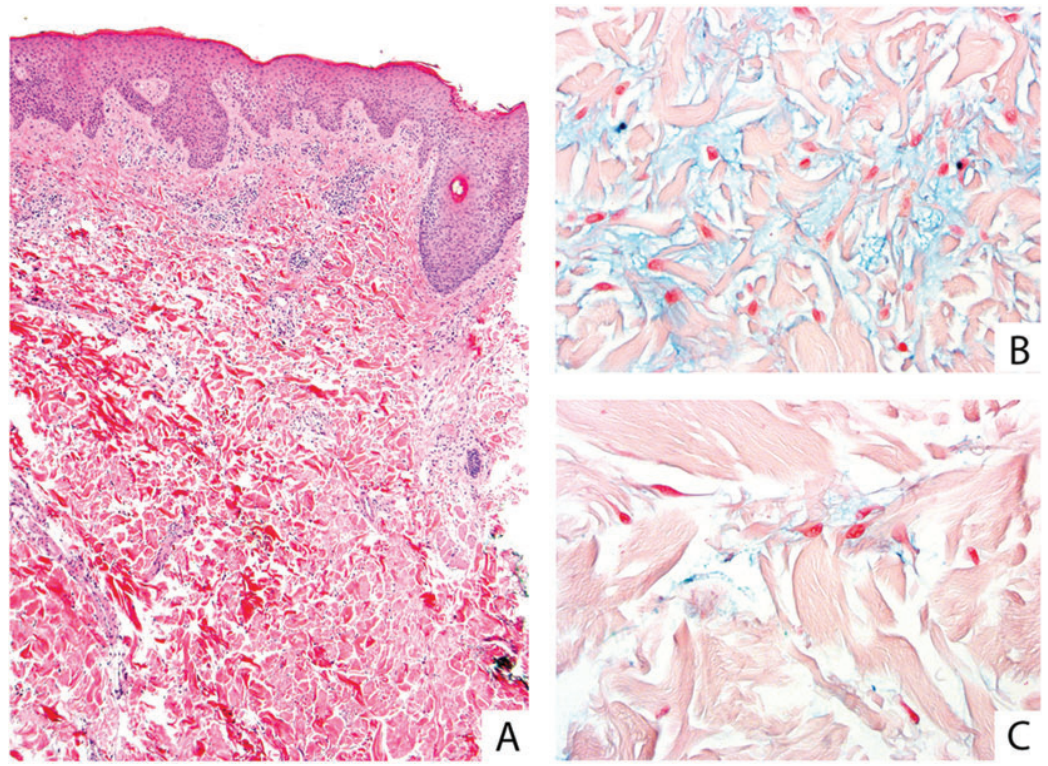

Fig. 3. An example case of eczematous dermatitis. A) Low magnification shows epidermal spongiosis and a mild superficial perivascular lymphocytic infiltrate. B) Abundant mucin is present in the superficial reticular dermis of this example. C) Only scant mucin is present in the deep reticular dermis. [hematoxylin-eosin, original magnification $\times 40$ (A); alcian blue, original magnifications $\times 400(\mathrm{~B}$ and $\mathrm{C})$ ]

study. As both superficial and deep reticular dermis yielded similar results, and four biopsy specimens were devoid of deep reticular dermis, the superficial reticular dermis is considered sufficient for the evaluation of mucin deposition. While the mean combined scores obtained from both the superficial and the deep reticular dermis are highly comparable, it is clear that the presence of moderate or abundant amount of mucin in the deep reticular dermis gives the highest specificity for LE. This is in agreement with common belief that deep dermal mucin is more specific than superficial dermal mucin.

Tumid LE is characterized by abundant dermal mucin deposition typically exceeding the amount seen in other LE subtypes, although no objective quantification has been reported to our knowledge. Our study included 22 cases 

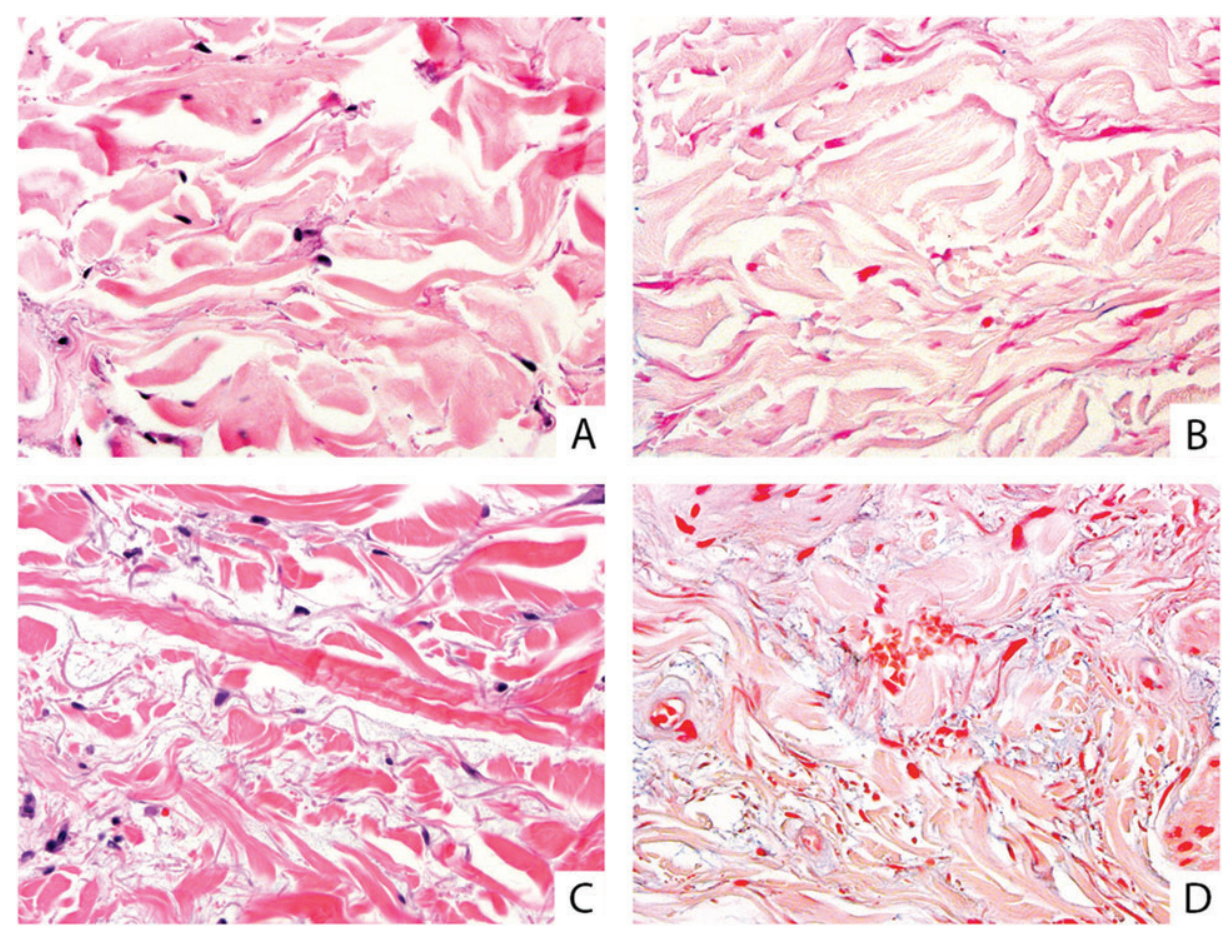

Fig. 4. Dermal mucin in non-photodamaged skin versus photodamaged skin. A and B) An example of non-photodamaged skin on the calf taken from the tip of a melanoma excision shows no mucin. C and D) Photodamaged skin on the scalp taken from the tip of a melanoma excision shows moderate amount of mucin. [hematoxylin-eosin (A and C) and alcian blue (B and D), original magnifications $\times 400]$

Table 3. Sensitivities and specificities of dermal mucin for lupus erythematosus against other dermatitides

\begin{tabular}{|c|c|c|c|c|c|c|}
\hline & \multicolumn{4}{|c|}{ Superficial reticular dermis } & \multirow{2}{*}{\multicolumn{2}{|c|}{$\begin{array}{l}\text { Deep reticular dermis } \\
\text { Combined score } \geq 4\end{array}$}} \\
\hline & \multicolumn{2}{|c|}{ Combined score $\geq 4$} & \multicolumn{2}{|c|}{ Combined score $\geq 5$} & & \\
\hline & Sensitivity (\%) & Specificity (\%) & Sensitivity (\%) & Specificity (\%) & Sensitivity (\%) & Specificity (\%) \\
\hline LE & 74.3 & - & 60.0 & - & 34.3 & - \\
\hline LE vs. all other dermatitides & - & 64.3 & - & 85.7 & - & 93.9 \\
\hline LE vs. GVHD & - & 87.5 & - & 87.5 & - & 100 \\
\hline LE vs. EM/FDE & - & 77.8 & - & 88.9 & - & 100 \\
\hline LE vs. LP & - & 93.3 & - & 100.0 & - & 100 \\
\hline LE vs. PMLE & - & 27.3 & - & 63.6 & - & 81.8 \\
\hline LE vs. urticaria & - & 50.0 & - & 75.0 & - & 100 \\
\hline LE vs. eczematous & - & 37.5 & - & 87.5 & - & 100 \\
\hline LE vs. psoriasis & - & 63.6 & - & 90.9 & - & 90.0 \\
\hline
\end{tabular}

EM, erythema multiforme; FDE, fixed drug eruption; GVHD, graft vs. host disease; LE, lupus erythematosus; LP, lichen planus; PMLE, polymorphous light eruption.

of non-tumid LE and 13 cases of tumid LE in order to avoid over-representation of the latter. As expected, tumid LE showed the highest mean scores in the reticular dermis in this study. Of the non-tumid subtypes, subacute cutaneous LE and discoid LE tend to exhibit greater amounts of mucin than systemic LE, although the small sample sizes preclude conclusive comparison.

Our data show that scant to moderate amount of dermal mucin can be seen in a variety of dermatitides besides LE, including those with spongiotic, psoriasiform, interface and/or perivascular tissue reaction patterns. While this creates little confusion in spongiotic and psoriasiform dermatitides, dermal mucin may present a diagnostic challenge when an interface process or a perivascular lymphocytic infiltrate is observed in conjunction with any clinical suspicion for LE. In this study, LE shows statistically higher mean combined mucin scores in the reticular dermis compared to all other dermatitides except PMLE and eczematous dermatitis. Of 
all the non-LE dermatitides examined, PMLE, eczematous dermatitis and urticaria exhibit the greatest amounts of dermal mucin. Using dermal mucin as an independent factor, a combined score of 5 and above - corresponding to abundant mucin on $\mathrm{H} \& \mathrm{E}$ and/or $\mathrm{AB}$ stains - is required to distinguish these three entities from LE.

Based on our results, distinction between PMLE and tumid LE proves to be most problematic. Both entities present clinically as erythematous papules and plaques on sun-exposed skin. ${ }^{20,21}$ Histopathologically, both are characterized by a superficial to deep perivascular lymphocytic infiltrate. While dermal mucin is another key feature of tumid LE, ${ }^{22}$ our data show for the first time that it is also a common finding in PMLE. This holds true for all three dermal compartments examined. To add to this diagnostic pitfall, a previous study has shown that marked papillary dermal edema (another characteristic feature of PMLE) may also be observed in acute cutaneous LE and discoid LE. ${ }^{23}$ In challenging cases, CD123 immunohistochemistry may serve as a useful discriminator, as it highlights plasmacytoid dendritic cells which are present in greater numbers and often in clusters in LE but not in PMLE. ${ }^{24,25}$

Interestingly, we also observed considerable amount of dermal mucin in eczematous dermatitis. Although the mean scores of eczematous dermatitis were lower than those of LE, the differences did not reach statistical significance. Morphologic distinction between eczematous dermatitis and LE usually poses little diagnostic challenge, as spongiosis is not typically seen in LE, and interface change is not a feature of eczematous dermatitis. A potentially problematic scenario would be an eczematous drug reaction, in which other minor reaction patterns including interface change may be observed. Recognition of mucin deposition as a common finding in eczematous dermatitis is therefore important in avoiding overdiagnosis of LE in this setting.

Mucin deposition in urticaria presents another potential diagnostic dilemma. Classic urticaria is characterized by a scant perivascular and interstitial neutrophilic infiltrate. Such reaction pattern has also been described in association with systemic LE as well as with other autoimmune diseases under different terminologies, including 'neutrophilic urticarial dermatosis', ${ }^{26}$ 'non-bullous neutrophilic LE', 27 'systemic LE-associated neutrophilic dermatosis', ${ }^{28}$ 'autoimmunity-related neutrophilic dermatosis', ${ }^{29}$ among others. Dermal mucin was observed in a subset of cases in various series, although the amount of mucin was not specified..$^{27-29}$ None of our patients with urticaria had any known history or clinical suspicion for LE. Our urticaria cases also lacked other typical features of systemic LE-associated neutrophilic dermatosis, such as basal vacuolar change along the dermoepidermal junction and prominent leukocytoclasis. ${ }^{26-29}$ Nevertheless, careful clinicopathologic correlation is required in the evaluation of urticarial dermatosis especially in the presence of dermal mucin, in order to exclude a paucicellular neutrophilic dermatosis associated with systemic LE.

Other interface dermatitides examined in this study (GVHD, EM/FDE and LP) displayed significantly less dermal mucin compared to LE. A combined score of 4 and above (at least moderate amount on both $\mathrm{H} \& \mathrm{E}$ and $\mathrm{AB}$ stains) in the superficial reticular dermis provides reasonable specificities in distinguishing these conditions from LE. On the other hand, the difference between LE and psoriasiform dermatitis showed narrower margin. Unlike interface dermatitides, however, psoriasis can be easily distinguished from LE based on its other features such as psoriasiform hyperplasia, hypogranulosis and confluent neutrophilic parakeratosis.

Using solar elastosis as an indicator of photodamage, we found increased deposition of dermal mucin in photodamaged normal skin compared to non-photodamaged normal skin. The difference is statistically significant using both combined $\mathrm{H} \& \mathrm{E}+\mathrm{AB}$ scores as well as $\mathrm{AB}$ scores alone; the latter argues against overinterpretation of solar elastosis on $\mathrm{H} \& \mathrm{E}$ as mucin deposition. While this may be common knowledge, our study provides the first objective proof of this phenomenon to our knowledge. We hypothesize that increased mucin in this setting is a result of degenerative change associated with photodamage, and possibly a failed reparative attempt. We also suggest that in photodamaged skin, the presence of increased dermal mucin is less reliable in distinguishing LE from other dermatitides than in nonphotodamaged skin.

In conclusion, this study confirms increased deposition of dermal mucin in cutaneous LE compared to other common dermatitides, although many non-LE conditions may also display scant to moderate amount of mucin. While histopathologic evaluation for dermal mucin remains key to the diagnosis of LE, its specificity as an independent factor is limited when 
present in low quantity or in a background of significant photodamage. Recognition of these findings will potentially avoid overdiagnosis of LE and inappropriate treatment. It should be emphasized that accurate diagnosis of cutaneous LE entails careful evaluation for all histopathologic features and adequate clinicopathologic correlation.

\section{References}

1. Montgomery H, Prunieras M. Histopathology of cutaneous lesions in systemic lupus erythematosus. AMA Arch Derm 1956; 74 : 177.

2. Weigand DA, Burgdorf WH, Gregg LJ. Dermal mucinosis in discoid lupus erythematosus. Report of two cases. Arch Dermatol 1981; 117: 735 .

3. Bangert JL, Freeman RG, Sontheimer RD, Gilliam JN. Subacute cutaneous lupus erythematosus and discoid lupus erythematosus. Comparative histopathologic findings. Arch Dermatol 1984; 120: 332.

4. Lanuti E, Miteva M, Romanelli P, Tosti A. Trichoscopy and histopathology of follicular keratotic plugs in scalp discoid lupus erythematosus. Int J Trichology 2012; 4: 36.

5. Schmitt V, Meuth AM, Amler S, et al. Lupus erythematosus tumidus is a separate subtype of cutaneous lupus erythematosus. Br J Dermatol 2010; 162: 64.

6. Yell JA, Mbuagbaw J, Burge SM. Cutaneous manifestations of systemic lupus erythematosus. Br J Dermatol 1996; 135: 355.

7. Taylor KR, Gallo RL. Glycosaminoglycans and their proteoglycans: host-associated molecular patterns for initiation and modulation of inflammation. FASEB J 2006; 20: 9 .

8. Chang LM, Maheshwari P, Werth S, et al. Identification and molecular analysis of glycosaminoglycans in cutaneous lupus erythematosus and dermatomyositis. J Histochem Cytochem 2011; 59: 336.

9. Almond A. Hyaluronan. Cell Mol Life Sci 2007; 64: 1591 .

10. Esko JD, Lindahl U. Molecular diversity of heparan sulfate. J Clin Invest 2001; 108: 169 .

11. Iozzo RV. Basement membrane proteoglycans: from cellar to ceiling. Nat Rev Mol Cell Biol 2005; 6: 646.

12. Matsuoka LY, Wortsman J, Dietrich JG, Kupchella CE. Glycosaminoglycans in histologic sections. Arch Dermatol 1987; 123: 862 .

13. Raman R, Sasisekharan V, Sasisekharan R. Structural insights into biological roles of protein-glycosaminoglycan interactions. Chem Biol 2005; 12: 267.

14. Xiao Z, Visentin GP, Dayananda KM, Neelamegham S. Immune complexes formed following the binding of anti-platelet factor 4 (CXCL4) antibodies to CXCL4 stimulate human neutrophil activation and cell adhesion. Blood 2008; 112: 1091.

15. Rachmilewitz J, Tykocinski ML. Differential effects of chondroitin sulfates A and $\mathrm{B}$ on monocyte and B-cell activation: evidence for B-cell activation via a CD44-dependent pathway. Blood 1998; 92: 223.

16. Fioravanti A, Collodel G. In vitro effects of chondroitin sulfate. Adv Pharmacol 2006; 53: 449 .

17. Pandya AG, Sontheimer RD, Cockerell CJ, Takashima A, Piepkorn M. Papulonodular mucinosis associated with systemic lupus erythematosus: possible mechanisms of increased glycosaminoglycan accumulation. J Am Acad Dermatol 1995; 32 (2 Pt 1): 199.

18. Wong T, McGrath JA, Navsaria H. The role of fibroblasts in tissue engineering and regeneration. Br J Dermatol 2007; 156: 1149.

19. Rongioletti F, Ghigliotti G, De Marchi R, Rebora A. Cutaneous mucinoses and HIV infection. Br J Dermatol 1998; 139: 1077.

20. Hsu S, Hwang LY, Ruiz H. Tumid lupus erythematosus. Cutis 2002; 69: 227.

21. Gruber-Wackernagel A, Byrne SN, Wolf P. Polymorphous light eruption: clinic aspects and pathogenesis. Dermatol Clin 2014; 32: 315 .

22. Kuhn A, Sonntag M, Ruzicka T, Lehmann P, Megahed M. Histopathologic findings in lupus erythematosus tumidus: review of
80 patients. J Am Acad Dermatol 2003; 48: 901.

23. Pincus LB, LeBoit PE, Goddard DS, Cho RJ, McCalmont TH. Marked papillary dermal edema-an unreliable discriminator between polymorphous light eruption and lupus erythematosus or dermatomyositis. J Cutan Pathol 2010; 37: 416.

24. Wackernagel A, Massone C, Hoefler G, Steinbauer E, Kerl H, Wolf P. Plasmacytoid dendritic cells are absent in skin lesions of polymorphic light eruption. Photodermatol Photoimmunol Photomed 2007; 23: 24.

25. Tomasini D, Mentzel T, Hantschke M, et al. Plasmacytoid dendritic cells: an overview of their presence and distribution in different inflammatory skin diseases, with special emphasis on Jessner's lymphocytic infiltrate of the skin and cutaneous lupus erythematosus. J Cutan Pathol 2010; 37: 1132.

26. Kieffer C, Cribier B, Lipsker D. Neutrophilic urticarial dermatosis: a variant of neutrophilic urticaria strongly associated with systemic disease. Report of 9 new cases and review of the literature Medicine (Baltimore) 2009; 88: 23.

27. Brinster NK, Nunley J, Pariser R, Horvath $B$. Nonbullous neutrophilic lupus erythematosus: a newly recognized variant of cutaneous lupus erythematosus. J Am Acad Dermatol 2012; 66: 92.

28. Larson AR, Granter SR. Systemic lupus erythematosus-associated neutrophilic dermatosis-an underrecognized neutrophilic dermatosis in patients with systemic lupus erythematosus. Hum Pathol 2014; 45: 598.

29. Saeb-Lima M, Charli-Joseph Y, Rodriguez-Acosta ED, Dominguez-Cherit J. Autoimmunity-related neutrophilic dermatosis: a newly described entity that is not exclusive of systemic lupus erythematosus. Am J Dermatopathol 2013; 35: 655 . 